CORRECTION

https://doi.org/10.1038/s41586-018-0302-0

\title{
Publisher Correction: Cryo-EM structure of human rhodopsin bound to an inhibitory $G$ protein
}

Yanyong Kang, Oleg Kuybeda, Parker W. de Waal,

Somnath Mukherjee, Ned Van Eps, Przemyslaw Dutka, X. Edward Zhou, Alberto Bartesaghi, Satchal Erramilli, Takefumi Morizumi, Xin Gu, Yanting Yin, Ping Liu, Yi Jiang, Xing Meng, Gongpu Zhao, Karsten Melcher, Oliver P. Ernst, Anthony A. Kossiakoff, Sriram Subramaniam \& H. Eric Xu

Correction to: Nature https://doi.org/10.1038/s41586-018-0215-y, published online 13 June 2018.

In the PDF version of this Article, owing to a typesetting error, an incorrect figure was used for Extended Data Fig. 5; the correct figure was used in the HTML version. This has been corrected online. 